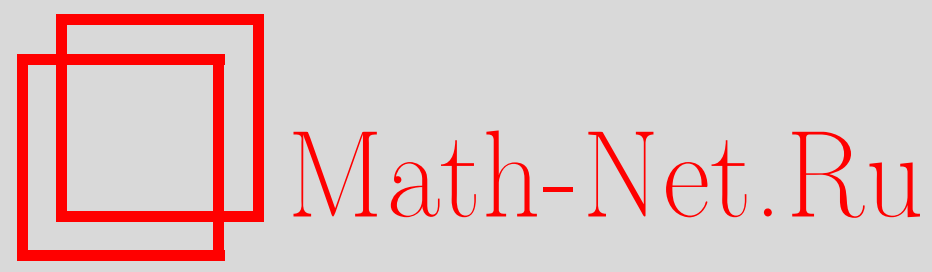

В. Г. Михайлов, О вероятности наличия в случайной последовательности цепочек с одинаковой структурой, Дискрет. матем., 2016, том 28, выпуск 3, 97-110

DOI: https://doi.org/10.4213/dm1385

Использование Общероссийского математического портала Math-Net.Ru подразумевает, что вы прочитали и согласны с пользовательским соглашением http://www . mathnet.ru/rus/agreement

Параметры загрузки:

IP : 3.81 .55 .215

26 апреля 2023 г., 13:08:30

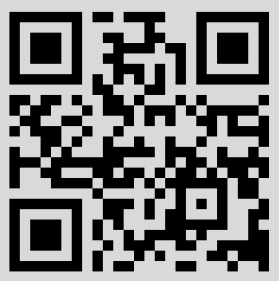




\section{О вероятности наличия \\ в случайной последовательности цепочек с одинаковой структурой}

() 2016 г. В. Г. Михайлов*

В работе получено асимптотическое выражение (с явно выписанной оценкой остаточного члена) для вероятности наличия в отрезке последовательности полиномиальных испытаний, управляемых цепью Маркова, цепочек с повторениями знаков, имеющих одинаковую структуру.

Исследование выполнено за счет гранта Российского научного фонда (проект № 14-50-00005).

Ключевые слова: полиномиальная схема, цепь Маркова, структура цепочки, эквивалентные цепочки

\section{1. Введение}

Под структурой цепочки $\left(x_{1}, \ldots, x_{s}\right)$ из элементов множества $\{1, \ldots, N\}$ понимается расположение одинаковых и разных букв в ней. Точнее, цепочки $\left(x_{1}, \ldots, x_{s}\right)$ и $\left(y_{1}, \ldots, y_{s}\right)$ обладают одинаковой структурой в том и только том случае, когда

$$
x_{i}=x_{j} \Longleftrightarrow y_{i}=y_{j}, \quad 1 \leqslant i<j \leqslant s .
$$

Это условие эквивалентно условию

$$
x_{i} \neq x_{j} \Longleftrightarrow y_{i} \neq y_{j}, \quad 1 \leqslant i<j \leqslant s .
$$

Совпадение структур цепочек $\left(x_{1}, \ldots, x_{s}\right)$ и $\left(y_{1}, \ldots, y_{s}\right)$ будет обозначаться $\left(x_{1}, \ldots, x_{s}\right) \sim\left(y_{1}, \ldots, y_{s}\right)$.

Цепочки называются эквивалентными цепочками, если найдется подстановка $\sigma$ : $(1, \ldots, N) \rightarrow(\sigma(1), \ldots, \sigma(N))$, при которой $\left(y_{1}, \ldots, y_{s}\right)=\left(\sigma\left(x_{1}\right), \ldots, \sigma\left(x_{s}\right)\right)$. Поэтому эквивалентными цепочками являются цепочки с одинаковой структурой и только они.

Пусть $\left(X_{t}\right)_{t=1}^{\infty}-$ некоторая последовательность случайных величин, принимающих значения из множества $\{1, \ldots, N\}$, а $Y_{t}=\left(X_{t}, \ldots, X_{t+s-1}\right)$ и

$$
\xi_{r}=\sum_{1 \leqslant i_{1} \ldots<i_{r} \leqslant n-s+1} I\left\{\left(Y_{i_{1}} \sim Y_{i_{2}} \sim \ldots \sim Y_{i_{r}}\right\}, \quad r=2, \ldots, n-s+1\right.
$$

* Место работы: Математический институт им. В. А. Стеклова Российской академии наук, е-mail: mikhail@mi.ras.ru 
где $I\{A\}$ обозначает индикатор случайного события $\{A\}$.

Свойства величин $\xi_{r}$ впервые были исследованы С. М. Буравлевым. В его статье [1] были получены достаточные условия сходимости распределения величины $\xi_{2}$ к распределению Пуассона для последовательности независимых случайных величин с равномерным распределением на множестве $\{1, \ldots, N\}$. В дальнейшем аналогичные и многие другие результаты были получены и в том случае, когда случайные величины $X_{t}$ независимы, но их распределение отлично от равномерного (см обзорную статью [2]). При этом выяснилось, что содержательные предельные теоремы для величин $\xi_{r}$ при $n, N \rightarrow \infty$ получаются только тогда, когда длина рассматриваемых $s$-цепочек больше или асимптотически равна $N$. Так, в [1] использовалось условие $s \geqslant N(\ln N+1)$. Оно, в частности, гарантирует, что в любой $s$-цепочке имеются повторения знаков.

Исследование распределений величин $\xi_{r}$ при $n, N \rightarrow \infty$ в ситуации, когда $s$ много меньше $N$, наталкивается на следующее препятствие. В этом случае большое число $s$-цепочек в последовательности не содержат повторений знаков. Все такие цепочки имеют одинаковую простую структуру (в [3] и [4] в этом случае использовался термин «тривиальная структура»). Так, при переходе к пределу по $n, N \rightarrow \infty$ и достаточно медленном росте $s$ главные члены величин $\xi_{r}$ оказываются детерминированными, что принципиально меняет поведение их распределений.

Естественным способом обойти это препятствие является исключение из рассмотрения цепочек с простой структурой. Для этого случайные величины $\xi_{r}$ модифицируются следующим образом. Пусть цепочка $y=(1,2, \ldots, s)$. Вместо $\xi_{r}$ предлагается рассматривать величины

$$
\xi_{r}^{*}=\sum_{1 \leqslant i_{1} \ldots<i_{r} \leqslant n-s+1} I\left\{\left(Y_{i_{1}} \sim Y_{i_{2}} \sim \ldots \sim Y_{i_{r}} \nsim y\right\}, \quad r=2, \ldots, n-s+1,\right.
$$

где запись $Y \not ⿻$ означает, что структуры цепочек $Y$ и $y$ не совпадают.

Особенность величин $\xi_{r}^{*}$ заключается в том, что они отличны от нуля только если выполнено событие

$E_{n, s}=\left\{\right.$ в отрезке $X_{1}, \ldots, X_{n}$ присутствуют $s$-цепочки, содержащие повторения знаков и имеющие при этом одинаковую структуру $\}$.

Кстати, из этих определений следует, что при $r=2,3, \ldots, n-s+1$

$$
\mathbf{P}\left\{\xi_{r}>0\right\}>\mathbf{P}\left\{\xi_{r}^{*}>0\right\} \geqslant \mathbf{P}\{E\} .
$$

Представляет интерес исследование вероятности события $E_{n, s}$. В [3] получена асимптотическая формула для вероятности этого события в случае последовательности полиномиальных испытаний. В настоящей работе мы распространяем этот результат на цепочки в последовательности полиномиальных испытаний, управляемых конечной цепью Маркова.

\section{2. Основные результаты}

Пусть $\left(X_{t}\right)_{t=1}^{\infty}-$ последовательность независимых не обязательно одинаково распределенных случайных величин, принимающих значения $1, \ldots, N$, распределения 
которых зависят от номера величины:

$$
\mathbf{P}\left\{X_{t}=k\right\}=p_{t, k}, \quad k=1, \ldots, N, \quad p_{t, 1}+\ldots+p_{t, N}=1 .
$$

Здесь $t=1,2, \ldots$

Пусть

$$
\begin{gathered}
\lambda_{k}=\sum_{i=1}^{n-s+1} \mathbf{P}\left\{X_{i}=X_{i+k}\right\}, \quad \Lambda=\sum_{k=1}^{s-1} \lambda_{k}, \\
\bar{P}_{2}=\max _{1 \leqslant i<j \leqslant n} \sum_{u=1}^{N} p_{i, u} p_{j, u}, \quad \bar{P}_{3}=\max _{1 \leqslant i<j<k \leqslant n} \sum_{u=1}^{N} p_{i, u} p_{j, u} p_{k, u} .
\end{gathered}
$$

Нас интересует вероятность события $E_{n, s}$, состоящего в том, что в отрезке $X(n)=$ $\left(X_{1}, \ldots, X_{n}\right)$ имеются $s$-цепочки с одинаковой «непростой» структурой.

Теорема 1. Пусть $n \geqslant s \geqslant 3, \bar{P}_{2}>0$. Тогда выполнено неравенство

$$
\begin{gathered}
\left|\mathbf{P}\left\{E_{n, s}\right\}-1+e^{-\Lambda} \prod_{k=1}^{s-1}\left(1+\lambda_{k}\right)\right|< \\
<\frac{(s-1)^{2}}{2}\left((n-s+1)\left((s+3) \bar{P}_{2}^{2}+7 \bar{P}_{3}\right)+\bar{P}_{2}\right) .
\end{gathered}
$$

Замечание 1. Теорема 1 является прямым обобщением теоремы 1 работы [3]. Она играет важную роль в нашем изложении. Ее утверждение и фрагменты доказательства будут использованы при выводе теоремы 2.

Перейдем к описанию основной задачи. Пусть теперь $\left(X_{t}\right)_{t=1}^{\infty}-$ последовательность случайных величин, принимающих значения $1, \ldots, N$, распределение которой определяется управляющей цепью $\left(Z_{t}\right)_{t=1}^{\infty}$ с состояниями $\{1, \ldots, M\}$ следующим образом: при всех $T$

$$
\mathbf{P}\left\{X_{t}=x_{t}, t=1, \ldots, T \mid\left\{Z_{t}=z_{t}, t=1, \ldots, T\right\}\right\}=\prod_{t=1}^{T} p_{x_{t}}^{\left(z_{t}\right)}
$$

где $x_{1}, \ldots, x_{T} \in\{1, \ldots, N\}, z_{1}, \ldots, z_{t} \in\{1, \ldots, M\}$, а набор $\left(p_{1}^{(m)}, \ldots, p_{N}^{(m)}\right)$ при каждом $m=1, \ldots, M$, задает вероятности $m$-й полиномиальной схемы с исходами $1, \ldots, N$. Такого рода последовательности принадлежат классу так называемых «скрытых марковских моделей». Проблематика этих моделей гораздо шире рассматриваемой нами задачи (см., например, [5]).

Будем считать, что цепь $\left(Z_{t}\right)_{t=1}^{\infty}$ является стационарной, неразложимой и непериодической с матрицей переходных вероятностей $\mathbb{Q}=\left\|q_{a, b}\right\|$, стационарными вероятностями $q_{1}, \ldots, q_{M}$ (предполагается, что все они положительны). Пусть $q_{v, w}^{(l)}-$ вероятность перехода цепи из $v$ в состояние $w$ за $l$ шагов. Как известно, найдутся такие числа $0<C<\infty$ и $\alpha>0$, что при всех $l \geqslant 1$

$$
\left|q_{v, w}^{(l)}-q_{w}\right| \leqslant C q_{w} e^{-\alpha l} .
$$


Пусть (эти определения аналогичны определениям (1))

$$
\begin{aligned}
\Lambda & =\sum_{k=1}^{s-1} \lambda_{k}, \quad \lambda_{k}=\sum_{i=1}^{n-s+1} \mathbf{P}\left\{X_{i}=X_{i+k}\right\}= \\
& =(n-s+1) \sum_{v=1}^{M} \sum_{w=1}^{M} \sum_{u=1}^{N} p_{u}^{(v)} p_{u}^{(w)} q_{v} q_{v, w}^{(k)},
\end{aligned}
$$

a

$$
\bar{P}_{2}=\max _{1 \leqslant i \leqslant j \leqslant M} \sum_{u=1}^{N} p_{u}^{(i)} p_{u}^{(j)}, \quad \bar{P}_{3}=\max _{1 \leqslant i \leqslant j \leqslant k \leqslant M} \sum_{u=1}^{N} p_{u}^{(i)} p_{u}^{(j)} p_{u}^{(k)} .
$$

Обратим внимание на то, что диапазоны параметров, по которым берутся максимумы в (7), отличаются от диапазонов в (2). Из (7) следует, что теперь $\bar{P}_{2}>0, \bar{P}_{3}>0$. Положим

$$
\Sigma=\sum_{k=1}^{s-1} \min \left\{1, \frac{1}{\sqrt{\lambda_{k}}}\right\} \sqrt{(2 k+1)(n-s+1) \bar{P}_{2}^{2}+\frac{C \lambda_{k}^{2} e^{-\alpha k}}{(n-s+1)\left(1-e^{-\alpha}\right)}} .
$$

Теорема 2. Пусть стационарная марковкая цепь $\left(Z_{t}\right)_{t=1}^{\infty}$ является неразложимой и непериодической с матрищей переходных вероятностей $\mathbb{Q}=\left\|q_{a, b}\right\|$, стационарными вероятностями $q_{1}, \ldots, q_{M}$ (предполагается, что все они положительнъ), a распределение последовательности $\left(X_{t}\right)_{t=1}^{\infty}$ определено формулой (4). Тогда при $n \geqslant s \geqslant 3$ для вероятности события $E_{n, s}$ выполнено неравенство

$$
\begin{gathered}
\left|\mathbf{P}\left\{E_{n, s}\right\}-1+e^{-\Lambda} \prod_{k=1}^{s-1}\left(1+\lambda_{k}\right)\right|< \\
<\frac{(s-1)^{2}}{2}\left((n-s+1)\left((s+3) \bar{P}_{2}^{2}+7 \bar{P}_{3}\right)+\bar{P}_{2}\right)+\Sigma .
\end{gathered}
$$

Следствие 1. Пусть выполнены условия теоремы $1, N, n \rightarrow \infty$, а параметр $s \geqslant 3$ и наборы вероятностей полиномиальных схем меняются так, что

$$
s^{2} n\left(s \bar{P}_{2}^{2}+\bar{P}_{3}\right) \rightarrow 0
$$

Тогда

$$
\begin{gathered}
\mathbf{P}\left\{E_{n, s}\right\}=1-e^{-\Lambda} \prod_{k=1}^{s-1}\left(1+\lambda_{k}\right)+O\left(s^{2} n \bar{P}_{3}+\left(s^{3 / 2} n^{1 / 2}+s^{2}\right) \bar{P}_{2}\right)= \\
=1-e^{-\Lambda} \prod_{k=1}^{s-1}\left(1+\lambda_{k}\right)+o(1) .
\end{gathered}
$$

В случае, когда $s \rightarrow \infty$, главный член асимптотики в (11) может быть выражен по-другому. Введем обозначение

$$
\mathbb{P}_{2}=\sum_{u=1}^{N}\left(\sum_{v=1}^{M} p_{u}^{(v)} q_{v}\right)^{2}
$$


Следствие 2. Пусть выполнены условия следствия $1, n>s \rightarrow \infty$. Тогда

$$
1-\mathbf{P}\left\{E_{n, s}\right\}=\exp \left\{-\frac{1}{2} s n^{2} \mathbb{P}_{2}^{2}\left(1+O\left(\frac{1}{s}+n \mathbb{P}_{2}\right)\right)\right\} .
$$

Если при этом

$$
n \sqrt{s} \mathbb{P}_{2} \rightarrow \varkappa \in(0, \infty)
$$

mo

$$
\mathbf{P}\left\{E_{n, s}\right\} \rightarrow 1-e^{-\varkappa^{2} / 2}
$$

\section{3. Доказательство теоремы 1}

В этом разделе $\left(X_{t}\right)_{t=1,2, \ldots}$ - последовательность независимых случайных величин, принимающих значения $1, \ldots, N$ с вероятностями $p_{t, 1}, \ldots, p_{t, N}$ соответственно, $t=$ $1,2, \ldots$

Будем говорить, что $s$-цепочка является цепочкой первого типа, если в ее записи имеется только одно повторение знака, а значит, используются ровно $s-1$ различных знаков. Если в записи $s$-цепочки использовано $s-2$ или меньше знаков, то назовем ее цепочкой второго типа.

Наряду с событием $E_{n, s}$, состоящим в том, что в отрезке $X(n)=\left(X_{1}, \ldots, X_{n}\right)$ имеются $s$-цепочки с одинаковой «непростой» структурой, рассмотрим события

$D_{1}=\{$ в отрезке $X(n)$ присутствуют $s$-цепочки второго типа $\}$,

$D_{2}=\left\{\right.$ в отрезке $X_{n-s+2}, \ldots, X_{n}$ имеется повторение знаков $\}$.

Определим случайные величины

$$
\eta_{k}=\sum_{i=1}^{n-s+1} I\left\{X_{i}=X_{i+k}\right\}, \quad k=1, \ldots, s-1,
$$

и событие

$$
E_{n, s}^{\prime}=\left\{\eta_{1} \geqslant 2\right\} \cup \ldots \cup\left\{\eta_{s-1} \geqslant 2\right\}
$$

Так как

$$
E_{n, s} \backslash\left(D_{1} \cup D_{2}\right)=E_{n, s}^{\prime} \backslash\left(D_{1} \cup D_{2}\right),
$$

то

$$
\left|\mathbf{P}\left\{E_{n, s}\right\}-\mathbf{P}\left\{E_{n, s}^{\prime}\right\}\right| \leqslant \mathbf{P}\left\{D_{1} \cup D_{2}\right\}=\mathbf{P}\left\{D_{1} \backslash D_{2}\right\}+\mathbf{P}\left\{D_{2}\right\} .
$$

Лемма 1. Пусть $n \geqslant s \geqslant 3$. Тогда выполнены неравенства

$$
\begin{aligned}
\mathbf{P}\left\{D_{1} \backslash D_{2}\right\} \leqslant & \frac{1}{2}(n-s+1)(s-1)_{2}\left((s-3) \bar{P}_{2}^{2}+\bar{P}_{3}\right), \\
& \mathbf{P}\left\{D_{2}\right\} \leqslant \frac{1}{2}(s-1)_{2} \bar{P}_{2}^{2} .
\end{aligned}
$$

Здесь и далее используется обозначение $(m)_{2}=m(m-1)$.

Доказательство леммы 1 лишь в деталях отличается от доказательства аналогичного утверждения в случае, когда вероятности $p_{t, 1}, \ldots, p_{t, N}$ не зависят от номера испытания $t$ (лемма 1 в [3]). Поэтому мы его не приводим. 
Пусть $\pi_{1}, \ldots, \pi_{s-1}-$ независимые случайные величины, имеющие распределения Пуассона с параметрами $\lambda_{1}, \ldots, \lambda_{s-1}$ (см. (1)) соответственно.

Расстояние по вариации между распределениями $\mathcal{L}(\xi)$ и $\mathcal{L}(\zeta)$ случайных величин $\xi$ и $\zeta$ будем обозначать через $\rho(\mathcal{L}(\xi), \mathcal{L}(\zeta))$. Напомним, что для распределений на конечном (или счетном) множестве $Y$

$$
\rho(\mathcal{L}(\xi), \mathcal{L}(\zeta))=\frac{1}{2} \sum_{y \in Y}|\mathbf{P}\{\xi=y\}-\mathbf{P}\{\zeta=y\}| .
$$

Лемма 2. Для расстояния по вариации между распределениями случайных векторов $\eta=\left(\eta_{1}, \ldots, \eta_{s-1}\right)$ u $\pi=\left(\pi_{1}, \ldots, \pi_{s-1}\right)$ выполнено неравенство

$$
\rho(\mathcal{L}(\eta), \mathcal{L}(\pi))<\left(3(s-1)_{2}+1\right)(n-s+1)\left(\bar{P}_{2}^{2}+\bar{P}_{3}\right) .
$$

Доказательство (леммы 2). Рассмотрим наборы случайных величин

$$
W=\left(I\left\{X_{t}=X_{t+k}\right\}, t=1, \ldots, n-s+1, k=1, \ldots, s-1\right)
$$

и

$$
\Pi=(\pi(t, k), t=1, \ldots, n-s+1, k=1, \ldots, s-1),
$$

где $\pi(t, k)$ - независимые случайные величины, $\pi(t, k)$ имеет распределение Пуассона с параметром $\mathbf{P}\left\{X_{t}=X_{t+k}\right\}$. Случайный вектор $\eta=\left(\eta_{1}, \ldots, \eta_{s-1}\right)$ является функцией вектора $W$, которая задается формулой (16) (будем использовать обозначение $\eta=F(W))$. Распределение случайного вектора $\pi=\left(\pi_{1}, \ldots, \pi_{s-1}\right)$, со своей стороны, совпадает с распределением вектора $F(\Pi)$. Поэтому из свойств расстояния по вариации получаем неравенство

$$
\rho(\mathcal{L}(\eta), \mathcal{L}(\pi)) \leqslant \rho(\mathcal{L}(W), \mathcal{L}(\Pi))
$$

Покажем, что

$$
\rho(\mathcal{L}(W), \mathcal{L}(\Pi))<\left(3(s-1)_{2}+1\right)(n-s+1)\left(\bar{P}_{2}^{2}+\bar{P}_{3}\right) .
$$

Воспользуемся тем, что случайные события $\left\{X_{t}=X_{t+k}\right\}$ определяются независимыми случайными величинами. Согласно теореме $10 \mathrm{~A}$ книги [6] в этом случае

$$
\rho(\mathcal{L}(W), \mathcal{L}(\Pi)) \leqslant S_{1}+S_{2},
$$

где

$$
\begin{gathered}
S_{1}=\sum_{|\{t, t+k\} \cup\{u, u+l\}|=3} \mathbf{P}\left\{X_{t}=X_{t+k}\right\} \mathbf{P}\left\{X_{u}=X_{u+l}\right\}+\sum_{t, t+k} \mathbf{P}^{2}\left\{X_{t}=X_{t+k}\right\}, \\
S_{2}=\sum_{|\{t, t+k\} \cup\{u, u+l\}|=3} \mathbf{P}\left\{X_{t}=X_{t+k}, X_{u}=X_{u+l}\right\}
\end{gathered}
$$

(в общем случае цитируемая оценка имеет более сложный вид.)

Поэтому

$$
S_{1}<\left(3(s-1)_{2}+1\right)(n-s+1) \bar{P}_{2}^{2}, \quad S_{2} \leqslant 3(s-1)_{2}(n-s+1) \bar{P}_{3} .
$$

Из (24) и (25) следует (23). В свою очередь, из (23) и (22) следует оценка (21). Лемма 2 доказана. 
Согласно определениям

$$
\left|\mathbf{P}\left\{E_{n, s}^{\prime}\right\}-1+\prod_{k=1}^{s-1} \mathbf{P}\left\{\pi_{k} \leqslant 1\right\}\right| \leqslant \rho(\mathcal{L}(\eta), \mathcal{L}(\pi)) .
$$

Поэтому из соотношений (17) и лемм 1 и 2 вытекают оценки

$$
\begin{gathered}
\left|\mathbf{P}\left\{E_{n, s}\right\}-1+\prod_{k=1}^{s-1}\left(\left(1+\lambda_{k}\right) e^{-\lambda_{k}}\right)\right| \leqslant \\
\leqslant \mathbf{P}\left\{D_{1} \backslash D_{2}\right\}+\mathbf{P}\left\{D_{2}\right\}+\rho(\mathcal{L}(\eta), \mathcal{L}(\pi))< \\
\quad<\frac{1}{2}(n-s+1)(s-1)_{2}\left((s-3) \bar{P}_{2}^{2}+\bar{P}_{3}\right)+ \\
+\frac{1}{2}(s-1)_{2} \bar{P}_{2}+\left(3(s-1)_{2}+1\right)(n-s+1)\left(\bar{P}_{2}^{2}+\bar{P}_{3}\right)< \\
<\frac{1}{2}(n-s+1)(s-1)^{2}\left((s+3) \bar{P}_{2}^{2}+7 \bar{P}_{3}\right)+\frac{1}{2}(s-1)_{2} \bar{P}_{2}< \\
<\frac{(s-1)^{2}}{2}\left((n-s+1)\left((s+3) \bar{P}_{2}^{2}+7 \bar{P}_{3}\right)+\bar{P}_{2}\right) .
\end{gathered}
$$

Из (26) следует (3). Теорема 1 доказана.

\section{4. Доказательство теоремы 2}

Пусть $\left(X_{t}\right)_{t=1}^{\infty}-$ последовательность случайных величин, принимающих значения $1, \ldots, N$, распределение которой определяется траекторией управляющей цепи $Z_{t}$ с состояниями $\{1, \ldots, M\}$ по формуле (4).

Нас интересует расстояние $\rho(\mathcal{L}(\eta), \mathcal{L}(\pi))$, где $\pi=\left(\pi_{1}, \ldots, \pi_{s-1}\right)$ - вектор из независимых случайных величин, распределенных по закону Пуассона с параметрами $\lambda_{k}=\mathbf{E} \lambda_{k}\left(Z_{1}, \ldots, Z_{n}\right)$ (см. (17) и (28) ниже)).

Лемма 3. Для расстояния по вариации между распределениями случайньх векторов $\eta=\left(\eta_{1}, \ldots, \eta_{s-1}\right)$ u $\pi=\left(\pi_{1}, \ldots, \pi_{s-1}\right)$ выполнено неравенство

$$
\rho(\mathcal{L}(\eta), \mathcal{L}(\pi))<\left(3(s-1)_{2}+1\right)(n-s+1)\left(\bar{P}_{2}^{2}+\bar{P}_{3}\right)+\Sigma .
$$

Договоримся при доказательстве теоремы 2 использовать сокращенные записи $\bar{z}=\left(z_{1}, \ldots, z_{n}\right)$ и $\bar{Z}=\left(Z_{1}, \ldots, Z_{n}\right)$.

Доказательство (леммы 3). Для произвольного набора $z_{1}, \ldots, z_{n} \in\{1, \ldots, M\}$ через $\mathcal{P}(\bar{z})$ обозначим условное распределение вектора $\eta=\left(\eta_{1}, \ldots, \eta_{s-1}\right)$ при условии $\left\{Z_{t}=z_{t}, t=1, \ldots, n\right\}$. Введем вектор

$$
\pi(\bar{z})=\left(\pi_{1}(\bar{z}), \ldots, \pi_{s-1}(\bar{z})\right)
$$

из независимых случайных величин $\pi_{k}(\bar{z}), k=1, \ldots, s$, величина $\pi_{k}(\bar{z})$ имеет распределение Пуассона с параметрами

$$
\lambda_{k}(\bar{z})=\sum_{i=1}^{n-s+1} \sum_{u=1}^{N} p_{u}^{\left(z_{i}\right)} p_{u}^{\left(z_{i+k}\right)}, \quad k=1, \ldots, s .
$$


Непосредственно из леммы 2 вытекает следующее утверждение.

Лемма 4. При всех $z_{1}, \ldots, z_{n} \in\{1, \ldots, M\}$ выполнено неравенство

$$
\rho(\mathcal{L}(\eta(\bar{z})), \mathcal{L}(\pi(\bar{z})))<\left(3(s-1)_{2}+1\right)(n-s+1)\left(\bar{P}_{2}^{2}+\bar{P}_{3}\right) .
$$

Так как неравенство (29) выполнено для всех $z_{1}, \ldots, z_{n} \in\{1, \ldots, M\}$, то из леммы 4 следует, что

$$
\rho(\mathcal{L}(\eta), \mathcal{L}(\pi(\bar{Z})))<\left(3(s-1)_{2}+1\right)(n-s+1)\left(\bar{P}_{2}^{2}+\bar{P}_{3}\right) .
$$

Здесь под $\mathcal{L}(\pi(\bar{Z}))$ понимается смесь распределений $\mathcal{L}(\pi(\bar{z}))$ по распределениям отрезка цепи $\bar{Z}$ :

$$
\mathbf{P}\{\pi(\bar{Z})=m\}=\sum_{\bar{z} \in\{1, \ldots, M\}^{n}} \frac{\lambda^{m}(\bar{z})}{m !} e^{-\lambda(\bar{z})} \mathbf{P}\{\bar{Z}=\bar{z}), \quad m=0,1, \ldots
$$

Перейдем к построению оценки для $\rho(\mathcal{L}(\pi(\bar{Z})), \mathcal{L}(\pi))$. Введем обозначение

$$
R_{k}(\bar{z})=\rho\left(\mathcal{L}\left(\pi_{k}(\bar{z})\right), \mathcal{L}\left(\pi_{k}\right)\right), \quad k=1, \ldots, s-1,
$$

тогда

$$
\mathbf{E} R_{k}(\bar{Z})=\sum_{z_{1}, \ldots, z_{n}} \rho\left(\mathcal{L}\left(\pi_{k}(\bar{z})\right), \mathcal{L}\left(\pi_{k}\right)\right) \mathbf{P}\left\{Z_{1}=z_{1}, \ldots, Z_{n}=z_{n}\right\} .
$$

Лемма 5. При всех $z_{1}, \ldots, z_{n} \in\{1, \ldots, M\}$ выполнено неравенство

$$
\rho\left(\mathcal{L}(\pi(\bar{Z}), \mathcal{L}(\pi)) \leqslant \sum_{k=1}^{s-1} \mathbf{E} R_{k}(\bar{Z}) .\right.
$$

Доказательство (леммы 5). Напомним, что $\pi_{1}, \ldots, \pi_{s-1}-$ независимые случайные величины, распределенные по закону Пуассона с параметрами $\lambda_{k}=\mathbf{E} \lambda_{k}(\bar{Z})$, $k=1, \ldots, s-1$. Обозначим

$$
\pi^{(k)}(\bar{Z})=\left(\pi_{1}, \ldots, \pi_{k}, \pi_{k+1}(\bar{Z}), \ldots, \pi_{s-1}(\bar{Z})\right), \quad k=1, \ldots, s-2 .
$$

Из свойств расстояния по вариации получаем

$$
\begin{gathered}
\rho\left(\mathcal{L}(\pi(\bar{Z}), \mathcal{L}(\pi)) \leqslant \rho\left(\mathcal{L}(\pi(\bar{Z})), \mathcal{L}\left(\pi^{(1)}(\bar{Z})\right)\right)+\right. \\
+\sum_{k=2}^{s-2} \rho\left(\mathcal{L}\left(\pi^{(k-1)}(\bar{Z})\right), \mathcal{L}\left(\pi^{(k)}(\bar{Z})\right)\right)+\rho\left(\mathcal{L}\left(\pi^{(s-2)}(\bar{Z})\right), \mathcal{L}\left(\pi_{1}, \ldots, \pi_{s-1}\right)\right) .
\end{gathered}
$$

Все слагаемые в правой части (33) оцениваются одинаково. Покажем, как это делается, на примере первого слагаемого. Обозначим $\pi^{\prime}(\bar{Z})=\left(\pi_{2}(\bar{Z}), \ldots, \pi_{s-1}(\bar{Z})\right)$, $l^{\prime}=\left(l_{2}, \ldots, l_{s-1}\right)$. Из определений и формулы $(20)$ следует, что

$$
\rho\left(\mathcal{L}(\pi(\bar{Z})), \mathcal{L}\left(\pi^{(1)}(\bar{Z})\right)\right)=
$$




$$
\begin{aligned}
& \left.=\frac{1}{2} \sum_{k=0}^{\infty} \sum_{l_{2}}^{\infty} \ldots \sum_{l_{s-1}}^{\infty} \mid \mathbf{P}\left\{\pi(\bar{Z})=\left(k, l^{\prime}\right)\right\}-\mathbf{P}\left\{\pi_{1}=k\right\} \mathbf{P}\left\{\pi^{\prime}(\bar{Z})\right)=l^{\prime}\right\} \mid< \\
& \quad<\frac{1}{2} \sum_{k=0}^{\infty} \sum_{l^{\prime} \in\{0,1, \ldots\}^{s-2}} \mid \sum_{z_{t}=1, t=1, \ldots, n}^{M} \mathbf{P}\left\{Z_{t}=z_{t}, t=1, \ldots, n\right\} \times \\
& \times\left(\mathbf{P}\left\{\pi_{1}(\bar{z})=k, \pi^{(1)}(\bar{z})=l^{\prime}\right\}-\mathbf{P}\left\{\pi_{1}=k\right\} \mathbf{P}\left\{\pi^{\prime}(\bar{z})=l^{\prime}\right\}\right) \mid= \\
& =\frac{1}{2} \sum_{k=0}^{\infty} \sum_{l^{\prime} \in\{0,1, \ldots\}^{s-2}} \mid \sum_{z_{t}=1, t=1, \ldots, n}^{M} \mathbf{P}\left\{Z_{t}=z_{t}, t=1, \ldots, n\right\} \mathbf{P}\left\{\pi^{\prime}(\bar{z})=l^{\prime}\right\} \times \\
& \left.\times \frac{1}{2} \sum_{k=0}^{\infty} \sum_{z_{t}=1, t=1, \ldots, n}^{M} \mathbf{P}\left\{\pi_{1}(\bar{z})=k\right\}-\mathbf{P}\left\{\pi_{1}=k\right\}\right) \mid \leqslant \\
& \times\left|\mathbf{P}\left\{\pi_{1}(\bar{z})=k\right\}-\mathbf{P}\left\{\pi_{1}=k\right\}\right|= \\
& =\frac{1}{2} \sum_{k=0}^{\infty} \mathbf{E}\left|\mathbf{P}\left\{\pi_{1}(\bar{Z})=k\right\}-\mathbf{P}\left\{\pi_{1}=k\right\}\right|=\mathbf{E} R_{1}(\bar{Z}) .
\end{aligned}
$$

Итак,

$$
\rho\left(\mathcal{L}(\pi(\bar{Z})), \mathcal{L}\left(\pi^{(1)}(\bar{Z})\right)\right) \leqslant \mathbf{E} R_{1}(\bar{Z}) .
$$

Остальные слагаемые аналогичным образом оцениваются величинами $\mathbf{E} R_{k}(\bar{Z})$, $k=2, \ldots, s-1$. Подставляя эти оценки в (33), получаем (32). Лемма 5 доказана.

Теперь надо оценить величины $\mathbf{E} R_{k}(\bar{Z})$. Для этого воспользуемся замечанием 1.1 .2 к теореме 1.С из книги [6], согласно которому расстояние по вариации между пуассоновскими распределениями с параметрами $\lambda$ и $\mu$ (обозначим их $\operatorname{Po}(\lambda)$ и $\operatorname{Po}(\mu)$ соответственно) удовлетворяет неравенству

$$
\rho(\operatorname{Po}(\lambda), \operatorname{Po}(\mu)) \leqslant \min \left\{1, \mu^{-1 / 2}\right\}|\lambda-\mu| .
$$

Применим эту оценку к функции $R_{k}(\bar{z})($ см. $(31))$, положив в ней $\mu=\mathbf{E} \lambda_{k}(\bar{Z})$. Тогда получим неравенство

$$
R_{k}(\bar{z})=\rho\left(\mathcal{L}\left(\pi_{k}(\bar{z})\right), \mathcal{L}\left(\pi_{k}\right)\right) \leqslant \min \left\{1,\left(\mathbf{E} \lambda_{k}(\bar{Z})\right)^{-1 / 2}\right\}\left|\lambda_{k}(\bar{z})-\mathbf{E} \lambda_{k}(\bar{Z})\right|,
$$

из которого следует оценка

$$
\begin{aligned}
\mathbf{E} R_{k}(\bar{Z}) \leqslant & \min \left\{1,\left(\mathbf{E} \lambda_{k}(\bar{Z})\right)^{-1 / 2}\right\} \mathbf{E}\left|\lambda_{k}(\bar{Z})-\mathbf{E} \lambda_{k}(\bar{Z})\right| \leqslant \\
& \leqslant \min \left\{1,\left(\mathbf{E} \lambda_{k}(\bar{Z})\right)^{-1 / 2}\right\} \sqrt{\mathbf{D} \lambda_{k}(\bar{Z})} .
\end{aligned}
$$


Оценим $\mathbf{D} \lambda_{k}(\bar{Z})$. Согласно (6)

$$
\begin{aligned}
\lambda_{k}=\mathbf{E} \lambda_{k}(\bar{Z}) & =\sum_{i=1}^{n-s+1} \sum_{v=1}^{M} \sum_{w=1}^{M} \sum_{u=1}^{N} p_{u}^{(v)} p_{u}^{(w)} \mathbf{P}\left\{Z_{i}=v, Z_{i+k}=w\right\}= \\
& =(n-s+1) \sum_{v=1}^{M} \sum_{w=1}^{M} \sum_{u=1}^{N} p_{u}^{(v)} p_{u}^{(w)} q_{v} q_{v, w}^{(k)},
\end{aligned}
$$

a

$$
\begin{aligned}
\mathbf{E} \lambda_{k}^{2}(\bar{Z})= & \sum_{i_{1}=1}^{n-s+1} \sum_{v_{1}=1}^{M} \sum_{w_{1}=1}^{M} \sum_{u_{1}=1}^{N} p_{u_{1}}^{\left(v_{1}\right)} p_{u_{1}}^{\left(w_{1}\right)} \sum_{i_{2}=1}^{n-s+1} \sum_{v_{2}=1}^{M} \sum_{w_{2}=1}^{M} \sum_{u_{2}=1}^{N} p_{u_{2}}^{\left(v_{2}\right)} p_{u_{2}}^{\left(w_{2}\right)} \times \\
& \times \mathbf{P}\left\{Z_{i_{1}}=v_{1}, Z_{i_{1}+k}=w_{1}, Z_{i_{2}}=v_{2}, Z_{i_{2}+k}=w_{2}\right\} .
\end{aligned}
$$

Для $l \geqslant 0$ обозначим через $J_{l}$ ту часть суммы в правой части $(37)$, для которой $i_{2}=i_{1}+l$. Тогда

$$
\mathbf{E} \lambda_{k}^{2}(\bar{Z})=J_{0}+2 \sum_{l=1}^{n-s} J_{l}
$$

В частности,

$$
\begin{aligned}
J_{0} & =\sum_{i=1}^{n-s+1} \sum_{v=1}^{M} \sum_{w=1}^{M} \sum_{u_{1}=1}^{N} p_{u_{1}}^{(v)} p_{u_{1}}^{(w)} \sum_{u_{2}=1}^{N} p_{u_{2}}^{(v)} p_{u_{2}}^{(w)} q_{v} q_{v, w}^{(k)} \leqslant \\
& \leqslant(n-s+1) \bar{P}_{2}^{2} \sum_{v=1}^{M} \sum_{w=1}^{M} q_{v} q_{v, w}^{(k)}=(n-s+1) \bar{P}_{2}^{2} .
\end{aligned}
$$

Пусть теперь $1 \leqslant l \leqslant k-1$. Тогда

$$
\begin{gathered}
J_{l}=\sum_{i=1}^{n-s-l+1} \sum_{v_{1}=1}^{M} \sum_{v_{2}=1}^{M} \sum_{u_{1}=1}^{N} p_{u_{1}}^{\left(v_{1}\right)} p_{u_{1}}^{\left(v_{3}\right)} \sum_{v_{3}=1}^{M} \sum_{v_{4}=1}^{M} \sum_{u_{2}=1}^{N} p_{u_{2}}^{\left(v_{2}\right)} p_{u_{2}}^{\left(v_{4}\right)} q_{v_{1}} q_{v_{1}, v_{2}}^{(l)} q_{v_{2}, v_{3}}^{(k-l)} q_{v_{3}, v_{4}}^{(l)} \leqslant \\
\leqslant(n-s-l+1) \bar{P}_{2}^{2} .
\end{gathered}
$$

Пусть $l=k$. Тогда

$$
\begin{aligned}
J_{1}= & \sum_{i=1}^{n-s} \sum_{v=1}^{M} \sum_{w=1}^{M} \sum_{u_{1}=1}^{N} p_{u_{1}}^{(v)} p_{u_{1}}^{(w)} \sum_{w_{2}=1}^{M} \sum_{u_{2}=1}^{N} p_{u_{2}}^{(w)} p_{u_{2}}^{\left(w_{2}\right)} q_{v} q_{v, w}^{(k)} q_{w, w_{2}}^{(k)} \leqslant \\
& \leqslant(n-s) \bar{P}_{2}^{2} \sum_{v=1}^{M} \sum_{w=1}^{M} \sum_{w_{2}=1}^{M} q_{v} q_{v, w}^{(k)} q_{w, w_{2}}^{(k)}=(n-s+1) \bar{P}_{2}^{2} .
\end{aligned}
$$

Наконец, пусть $k<l \leqslant n-s$. Тогда

$$
J_{l}=\sum_{i=1}^{n-s-l+1} \sum_{v_{1}=1}^{M} \sum_{v_{2}=1}^{M} \sum_{u_{1}=1}^{N} p_{u_{1}}^{\left(v_{1}\right)} p_{u_{1}}^{\left(v_{2}\right)} \sum_{v_{3}=1}^{M} \sum_{v_{4}=1}^{M} \sum_{u_{2}=1}^{N} p_{u_{2}}^{\left(v_{3}\right)} p_{u_{2}}^{\left(v_{4}\right)} q_{v_{1}} q_{v_{1}, v_{2}}^{(k)} q_{v_{2}, v_{3}}^{(l-k)} q_{v_{3}, v_{4}}^{k} \leqslant
$$




$$
\leqslant(n-s-l+1) \bar{P}_{2}^{2} .
$$

Но в этом случае удобнее воспользоваться свойством (5), согласно которому найдутся такие числа $0<C<\infty$ и $\alpha>0$, что при всех $l \geqslant 1$

$$
\left|q_{v, w}^{(l)}-q_{w}\right| \leqslant C q_{w} e^{-\alpha l}
$$

Поэтому

$$
\begin{gathered}
J_{l} \leqslant \sum_{i=1}^{n-s+1} \sum_{v_{1}=1}^{M} \sum_{v_{2}=1}^{M} \sum_{u_{1}=1}^{N} p_{u_{1}}^{\left(v_{1}\right)} p_{u_{1}}^{\left(v_{2}\right)} q_{v_{1}} q_{v_{1}, v_{2}}^{(k)} \sum_{v_{3}=1}^{M} \sum_{v_{4}=1}^{M} \sum_{u_{2}=1}^{N} p_{u_{2}}^{\left(v_{3}\right)} p_{u_{2}}^{\left(v_{4}\right)} q_{v_{3}} q_{v_{3}, v_{4}}^{(k)}\left(1+C e^{-\alpha(l-1)}\right)= \\
=(n-s-l+1)\left(\sum_{v_{1}=1}^{M} \sum_{v_{2}=1}^{M} \sum_{u=1}^{N} p_{u}^{\left(v_{1}\right)} p_{u}^{\left(v_{2}\right)} q_{v_{1}} q_{v_{1}, v_{2}}^{(k)}\right)^{2}\left(1+C e^{-\alpha(l-1)}\right) .
\end{gathered}
$$

Из (39) - (41) следует, что

$$
J_{0}+2 \sum_{l=1}^{k} J_{l} \leqslant(2 k+1)(n-s+1) \bar{P}_{2}^{2}
$$

В свою очередь, из (43) и (36) вытекают неравенства

$$
\begin{gathered}
\sum_{l=k+1}^{n-s} J_{l}<\left(\sum_{v_{1}=1}^{M} \sum_{v_{2}=1}^{M} \sum_{u=1}^{N} p_{u}^{\left(v_{1}\right)} p_{u}^{\left(v_{2}\right)} q_{v_{1}} q_{v_{1}, v_{2}}^{(k)}\right)^{2} \sum_{l=k+1}^{n-s}(n-s-l+1)\left(1+C e^{-\alpha(l-1)}\right)< \\
\quad<\frac{1}{2}\left(\left(\mathbf{E} \lambda_{k}(\bar{Z})\right)^{2}+\frac{C \lambda_{k}^{2}}{n-s+1} \sum_{l=k+1}^{n-s} e^{-\alpha(l-1)}\right) .
\end{gathered}
$$

Теперь из (38) и оценок (44), (45) следует, что

$$
\mathbf{E} \lambda_{k}^{2}(\bar{Z})<(2 k+1)(n-s+1) \bar{P}_{2}^{2}+\left(\mathbf{E} \lambda_{k}(\bar{Z})\right)^{2}+\frac{C \lambda_{k}^{2} e^{-\alpha k}}{(n-s+1)\left(1-e^{-\alpha}\right)} .
$$

Из (36) и (46) вытекает оценка для дисперсии:

$$
\mathbf{D} \lambda_{k}(\bar{Z})=\mathbf{E} \lambda_{k}^{2}(\bar{Z})-\left(\mathbf{E} \lambda_{k}(\bar{Z})\right)^{2}<(2 k+1)(n-s+1) \bar{P}_{2}^{2}+\frac{C \lambda_{k}^{2} e^{-\alpha k}}{(n-s+1)\left(1-e^{-\alpha}\right)} .
$$

Из (47) и (35) получаем, что

$$
\mathbf{E} R_{k}(\bar{Z})<\min \left\{1, \frac{1}{\sqrt{\lambda_{k}}}\right\} \sqrt{(2 k+1)(n-s+1) \bar{P}_{2}^{2}+\frac{C \lambda_{k}^{2} e^{-\alpha k}}{(n-s+1)\left(1-e^{-\alpha}\right)}},
$$

а из (48) и леммы 5 - оценку

$$
\rho(\mathcal{L}(\pi(\bar{Z}), \mathcal{L}(\pi))<\Sigma
$$


где, напомним,

$$
\Sigma=\sum_{k=1}^{s-1} \min \left\{1, \frac{1}{\sqrt{\lambda_{k}}}\right\} \sqrt{(2 k+1)(n-s+1) \bar{P}_{2}^{2}+\frac{C \lambda_{k}^{2} e^{-\alpha k}}{(n-s+1)\left(1-e^{-\alpha}\right)}} .
$$

Теперь из (30), (49) и неравенства треугольника следует неравенство (27). Лемма 3 доказана.

Перейдем непосредственно к доказательству теоремы 2. Будем следовать схеме доказательства теоремы 1 , используя те же самые обозначения, применяя их к последовательности $\left(X_{t}\right)_{t=1}^{\infty}$ с распределением (4).

При условии $Z_{t}=z_{t}, t=1, \ldots, n$, отрезок $\left(X_{t}\right)_{t=1}^{n}$ распределен как последовательность исходов неоднородной полиномиальной схемы. Поэтому из наших определений, неравенства (17) и леммы 1 следует, что

$$
\begin{gathered}
\left|\mathbf{P}\left\{E_{n, s}\right\}-\mathbf{P}\left\{E_{n, s}^{\prime}\right\}\right| \leqslant \max _{z_{1}, \ldots, z_{n}} \mathbf{P}\left\{D_{1} \cup D_{2} \mid Z_{t}=z_{t}, t=1, \ldots, n\right\} \leqslant \\
\leqslant \frac{1}{2}(n-s+1)(s-1)_{2}\left((s-3) \bar{P}_{2}^{2}+\bar{P}_{3}\right)+\mathbf{P}\left\{D_{2}\right\} .
\end{gathered}
$$

Далее вместо леммы 2 мы пользуемся леммой 3. Оценка леммы 3 отличается от оценки леммы 2 лишь присутствием дополнительного слагаемого $\Sigma$. Поэтому все дальнейшие рассуждения доказательства теоремы 2 отличаются от рассуждений доказательства теоремы 1 лишь присутствием в них этого слагаемого. Оно же добавляется к итоговой оценке. Поэтому (9) следует из (3). Теорема 2 доказана.

Замечание 2. Благодаря особенностям распределения (4) метод Чена - Стейна при доказательстве теоремы 2 удается использовать по другой схеме и с меньшими трудностями, чем при аналогичных исследованиях повторений в цепях Маркова (см., например, [7] - [10]).

\section{5. Доказательства следствий}

Доказательство (следствия 1). Из наших определений и формул (6), (8) следует, что оценкой первого слагаемого под знаком корня в выражении для $\Sigma$ является $O\left(s n \bar{P}_{2}^{2}\right)$, а второго $-O\left(n \bar{P}_{2}^{2}\right)$. Поэтому

$$
\Sigma=O\left(s \sqrt{s n \bar{P}_{2}^{2}}\right)=O\left(s^{3 / 2} n^{1 / 2} \bar{P}_{2}\right) .
$$

Подставив эту оценку в правую часть (9), получим, что выражение в правой части (9) допускает оценку

$$
O\left(s^{2} n\left(s \bar{P}_{2}^{2}+\bar{P}_{3}\right)+\left(s^{3 / 2} n^{1 / 2}+s^{2}\right) \bar{P}_{2}\right),
$$

которая при условии (10) превращается в оценку

$$
O\left(s^{2} n \bar{P}_{3}+\left(s^{3 / 2} n^{1 / 2}+s^{2}\right) \bar{P}_{2}\right) .
$$

С учетом этой оценки соотношение (11) вытекает из (9). Следствие 1 доказано. 
Доказательство (следствия 2). Введем обозначение

$$
\lambda_{\infty}=(n-s+1) \sum_{v=1}^{M} \sum_{w=1}^{M} \sum_{u=1}^{N} p_{u}^{(v)} p_{u}^{(w)} q_{v} q_{w}=(n-s+1) \mathbb{P}_{2} .
$$

Используя свойство (5), получаем неравенства

$$
\begin{gathered}
\lambda_{k}<\left(1+C e^{-\alpha k}\right) n \mathbb{P}_{2}, \quad k=1,2, \ldots, \\
\left|\lambda_{k}-\lambda_{\infty}\right|<C n \mathbb{P}_{2} e^{-\alpha k}, \quad\left|\lambda_{k}^{2}-\lambda_{\infty}^{2}\right|<C(2+C) n^{2} \mathbb{P}_{2}^{2} e^{-\alpha k}, \\
\sum_{k=1}^{s-1} \lambda_{k}^{3}<(1+C)^{3} s n^{3} \mathbb{P}_{2}^{3} .
\end{gathered}
$$

Следовательно,

$$
\begin{gathered}
\left|\sum_{k=1}^{s-1} \lambda_{k}^{2}-(s-1) \lambda_{\infty}^{2}\right|<C(2+C) n^{2} \mathbb{P}_{2}^{2} \sum_{k=1}^{s-1} e^{-\alpha k}=O\left(n^{2} \mathbb{P}_{2}^{2}\right), \\
\sum_{k=1}^{s-1} \lambda_{k}^{3}=O\left(s n^{3} \mathbb{P}_{2}^{3}\right) .
\end{gathered}
$$

С помощью оценок (52) и (53) получаем

$$
\begin{gathered}
e^{-\Lambda} \prod_{k=1}^{s-1}\left(1+\lambda_{k}\right)=\exp \left\{-\frac{1}{2} \sum_{k=1}^{s-1} \lambda_{k}^{2}+O\left(\sum_{k=1}^{s-1} \lambda_{k}^{3}\right)\right\}= \\
=\exp \left\{-\frac{1}{2}(s-1) \lambda_{\infty}^{2}+\frac{1}{2}\left((s-1) \lambda_{\infty}^{2}-\sum_{k=1}^{s-1} \lambda_{k}^{2}\right)+O\left(\sum_{k=1}^{s-1} \lambda_{k}^{3}\right)\right\} .
\end{gathered}
$$

Так как $s \rightarrow \infty$, то из (50), (52) - (54) вытекает (13).

Соотношение (15) вытекает из (13) и (14). Следствие 2 доказано.

Автор признателен А. М. Зубкову за полезные замечания.

\section{Список литературы}

1. Буравлев С. М., "Повторения с точностью до перестановок в последовательности независимых испытаний”, Дискретная математика, 11:1 (1999), 53-75; англ. пер.: Buravlev S. M., "Matchings up to permutations in sequences of independent trials", Discrete Math. Appl., 9:1 (1999), 53-78.

2. Михайлов В. Г., Шойтов А. М., "Структурная эквивалентность $s$-цепочек в случайных дискретных последовательностях", Дискретная математика, 15:4 (2003), 7-34; англ. пер.: Mikhailov V. G., Shoitov A. M., "Structural equivalence of $s$-tuples in random discrete sequences", Discrete Math. Appl., 13:6 (2003), 541-568.

3. Михайлов В. Г., "Об асимптотическом поведении вероятности наличия в последовательности эквивалентных цепочек с нетривиальной структурой”, Дискретная математика, 20:4 (2008), 113-119; англ. пер.: Mikhailov V. G., "On the asymptotic behaviour of the probability of existence of equivalent tuples with nontrivial structure in a random sequence", Discrete Math. Appl., 18:6 (2008), 563-568. 
4. Михайлов В. Г., Шойтов А. М., “О числах множеств эквивалентных цепочек в последовательности независимых случайных величин”, Математические вопросы криптографии, 4:1 (2013), 77-86.

5. Elliott R. J., Aggoun L., Moore J. B. Hidden Markov Models, N.-Y.: Springer-Verlag, 1995.

6. Barbour A. D., Holst L., Janson S., Poisson Approximation, Oxford University Press, Oxford, 1992.

7. Chryssaphinou O., Vaggelatou E., "Compound Poisson approximation for multiple runs in a Markov chain", Ann. Inst. Statist. Math., 54:2 (2002), 411-424.

8. Михайлов В. Г., Шойтов А. М., "О длинных повторениях цепочек в цепи Маркова", Дискретная математика, 26:3 (2014), 79-89; англ. пер.: Mikhailov V. G., Shoitov A. M., "On repetitions of long tuples in a Markov chain", Discrete Math. Appl., 25:5 (2015), 295-303.

9. Михайлов В. Г., Шойтов А. М., "Многократные повторения длинных цепочек в цепи Маркова", Математические вопросы криптографии, 6:3 (2015), 117-134.

10. Михайлов В. Г., “Оценки точности пуассоновской аппроксимации для распределения числа серии повторений длинных цепочек в цепи Маркова", Дискретная математика, 27:4 (2015), 67-78; англ. пер.: Mikhailov V. G., "Estimates of accuracy of the Poisson approximation for the distribution of number of runs of long string repetitions in a Markov chain", Discrete Math. Appl., 26:2 (2016), 105-113.

Статья поступила 15.06.2016. 